\title{
Nonlinear properties of left-handed metamaterials
}

\author{
Alexander A. Zharov ${ }^{1,2}$, Ilya V. Shadrivov ${ }^{1}$, and Yuri S. Kivshar ${ }^{1}$ \\ ${ }^{1}$ Nonlinear Physics Group, Research School of Physical Sciences and Engineering, \\ Australian National University, Canberra ACT 0200, Australia \\ 2 Institute for Physics of Microstructures, Russian Academy of Sciences, Nizhny Novgorod 603950, Russia
}

\begin{abstract}
We analyze nonlinear properties of microstructured materials with negative refraction, the socalled left-handed metamaterials. We consider a two-dimensional periodic structure created by arrays of wires and split-ring resonators embedded into a nonlinear dielectric, and calculate the effective nonlinear electric permittivity and magnetic permeability. We demonstrate that the hysteresis-type dependence of the magnetic permeability on the field intensity allows changing the material from left- to right-handed and back. These effects can be treated as the second-order phase transitions in the transmission properties induced by the variation of an external field.
\end{abstract}

PACS numbers: 41.20.Jb, 42.25.Bs, 78.20.Ci, 42.70.Qs

Recent theoretical studies [1, 2, 3] and experimental results [4, 5, 6] have shown the possibility to create novel types of microstructured materials which demonstrate the property of negative refraction. In particular, the composite materials created by arrays of wires and splitring resonators were shown to possess the negative real part of the magnetic permeability and dielectric permittivity for microwaves. These materials are often referred to as left-handed materials or materials with negative refraction. Properties of such materials were analyzed theoretically by Veselago long time ago [], but only recently they were demonstrated experimentally. As was shown by Veselago [], the left-handed materials possess a number of peculiar properties, including negative refraction for the interface scattering, inverse light pressure, reverse Doppler and Vavilov-Cherenkov effects.

So far, all properties of left-handed materials were studied in the linear regime of wave propagation when both magnetic permeability and dielectric permittivity of the material are assumed to be independent on the intensity of the electromagnetic field. However, any future effort in creating tunable structures where the field intensity changes the transmission properties of the composite structure would require the knowledge of nonlinear properties of such metamaterials which may be quite unusual. In this Letter we analyze, for the first time to our knowledge, nonlinear properties of left-handed metamaterials for the example of a lattice of the split-ring resonators and wires with a nonlinear dielectric. We show that the effective magnetic permeability depends on the intensity of the macroscopic magnetic field in a nontrivial way, allowing switching between the left- and right-handed materials by varying the field intensity. We believe that our findings may stimulate the future experiments in this field, as well as the studies of nonlinear effects in photonic crystals where the phenomenon of negative refraction is analyzed now very intensively [8, 9].

We consider a two-dimensional composite structure consisting of a square lattice of the periodic arrays of conducting wires and split-ring resonators (SRR), shown schematically in Fig. [1] We assume that the unit-cell size $d$ of the structure is much smaller then the wavelength of the propagating electromagnetic field and, for simplicity, we choose the single-ring geometry of a lattice of cylindrical SRRs. The results obtained for this case are qualitatively similar to more involved cases of double SRRs properties. This type of the microstructured materials has recently been suggested and built in order to create left-handed metamaterials with negative refraction in the microwave region [4].

Negative real part of the effective dielectric permittivity of such a composite structure appears due to the metallic wires whereas a negative sign of the magnetic permeability becomes possible due to the SRR lattice. As a result, these materials demonstrate the properties of negative refraction in the finite frequency band, $\omega_{0}<\omega<\min \left(\omega_{p}, \omega_{\| m}\right)$, where $\omega_{0}$ is the eigenfrequency of SRRs, $\omega_{\| m}$ is the frequency of the longitudinal magnetic plasmon, $\omega_{p}$ is an effective plasma frequency, and $\omega$ is an angular frequency of the propagating electromagnetic wave, $(\mathcal{E}, \mathcal{H}) \sim(\mathbf{E}, \mathbf{H}) \exp (i \omega t)$. The split-ring resonator can be described as an effective LC oscillator (see Ref. [10]) with capacitance of the SRR gap, as well as effective inductance, and resistance (see the upper insert in Fig. (1).

Nonlinear response of such a composite structure can be characterized by two different contributions. The first one is an intensity-dependent part of the effective dielectric permittivity of the infilling dielectric. For simplicity, we assume that the metallic structure is embedded into a nonlinear dielectric with permittivity that depends on the intensity of electric field in a general form, $\epsilon_{D}=\epsilon_{D}\left(|\mathbf{E}|^{2}\right)$. For detailed calculations presented below, we take the linear dependence that corresponds to the Kerr nonlinearity.

The second contribution into the nonlinear properties of the composite material comes from the lattice of resonators, since the SRR capacitance (and, therefore, the SRR eigenfrequency) depends on the strength of a local electric field in a narrow slot. Additionally, we can expect a nonlinear eigenfrequency detuning due to a resonant growth of the charge density at the edges of the SRR gap. The intensity of the local electric field in the SRR gap depends on the electromotive force in the resonator 


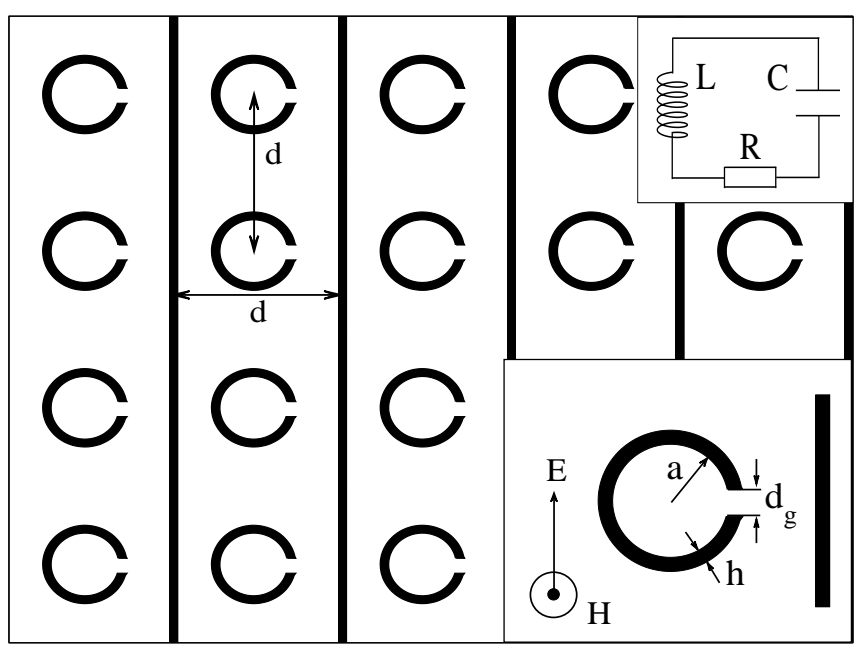

FIG. 1: Schematic of the composite metamaterial structure. Lower insert shows a unit cell of the periodic structure while the upper inset shows the SRR equivalent oscillator with the parameters used in the derivation.

loop, which is induced by the magnetic field. Therefore, the effective magnetic permeability $\mu_{\text {eff }}$ should depend on the macroscopic (average) magnetic field $\mathbf{H}$.

For the polarization shown in Fig. 1 (lower insert), the main contribution to the dielectric function is given by the array of wires. When the wire length is large enough, so that the frequency of the fundamental (dipole) mode of an individual wire becomes much less than $\omega$, only the resistance and inductance of the wires give a contribution into the lattice impedance. Hence, the Ohm law for the current can be written in the form,

$$
\mathbf{j}_{\omega} \approx \frac{\sigma}{1+i \omega \sigma S L_{w}} \mathbf{E}^{\prime}
$$

where $\mathbf{j}_{\omega}$ is the electric current density in the wire, $\mathbf{E}^{\prime}$ is the local electric field, $\sigma$ is the conductivity of the wire metal, $L_{w} \approx 2 c^{-2} \ln (d / r)(d \gg r)$ is the inductance of the wire per unit length, $c$ is the speed of light, $r$ is the wire radius, $S$ is the effective area of the wire crosssection, $S \approx \pi r^{2}$, for $\delta>r$ and $S \approx \pi \delta(2 r-\delta)$, for $\delta<r$, where $\delta=c / \sqrt{(2 \pi \sigma \omega)}$ is the thickness of the skin layer. The average current density in the unit sell can be written in the form,

$$
\left\langle\mathbf{j}_{\omega}\right\rangle=\frac{S}{d^{2}} \mathbf{j}_{\omega} .
$$

For the waves polarized along the wires the average macroscopic electric field $\mathbf{E}$ is approximately equal to the local field $\mathbf{E}^{\prime}$. Taking into account the general relation between the electric field $\mathbf{E}$ and the electric induction $\mathbf{D}$,

$$
\mathbf{D}=\epsilon_{D}\left(|\mathbf{E}|^{2}\right) \mathbf{E}+\frac{4 \pi}{i \omega}\left\langle\mathbf{j}_{\omega}\right\rangle,
$$

we can obtain the expression for the effective nonlinear dielectric permittivity

$$
\epsilon_{\mathrm{eff}}\left(|E|^{2}\right)=\epsilon_{D}\left(|E|^{2}\right)-\frac{\omega_{p}^{2}}{\omega\left(\omega-i \gamma_{\epsilon}\right)},
$$

where $\omega_{p} \approx(c / d)[2 \pi / \ln (d / r)]^{1 / 2}$ is the effective plasma frequency, and $\gamma_{\epsilon}=c^{2} / 2 \sigma S \ln (d / r)$. The second term in the right-hand side of Eq. (4) is in a full agreement with the earlier result obtained by Pendry and co-authors [1]. One should note that the low losses case, i.e. $\gamma_{\epsilon} \ll \omega$, corresponds to the condition $\delta \ll r$.

The analysis becomes more involved for calculating $a$ nonlinear magnetic response of the composite structure, which is determined by the intrinsic properties of the interacting nonlinear oscillators in the presence of an external periodic force. For the structure under consideration, the current induced in each resonator can be found as

$$
I=-i \pi a^{2}\left(\frac{\omega}{c}\right)\left|\mathbf{H}_{0}\right| Z^{-1},
$$

where $\mathbf{H}_{0}$ is the amplitude of the external field applied to SRR, $Z=i \omega L+R+(i \omega C)^{-1}$ is the SRR impedance, and other parameters are marked in Fig. [1 $a$ is the radius, $L, R$, and $C$ are inductance, resistance and capacitance, respectively. The amplitude of the electric field in the gap of SRR can be found approximately as follows

$$
\left|\mathbf{E}_{g}\right| \approx \frac{I}{i \omega C d_{g}}
$$

where $d_{g}$ is the size of the SRR gap. Nonlinear effects in Eqs. (5), (6) appear due to the capacitance $C$ which is proportional to $\epsilon_{D}\left(\left|E_{g}\right|^{2}\right)$. Therefore, Eq. (6) gives an implicit relation between the amplitude of the local electric field in the gap and the amplitude of the (external to SRR) magnetic field.

The relation between the local (microscopic) and average (macroscopic) magnetic fields can be obtained by averaging a local field over the volume of the unit cell.

$$
\langle\mathbf{B}\rangle=\langle\mathbf{H}\rangle+F \mathbf{H}^{\prime},
$$

where $\mathbf{H}^{\prime}$ is an additional magnetic field induced by the alternating external magnetic field $\mathbf{H}_{0}$ in cylindrical SRR, which determines magnetization of the composite, and $F=\pi a^{2} / d^{2}$.

The average macroscopic field can be also determined as follows,

$$
\mathbf{H} \equiv\langle\mathbf{H}\rangle=(1-F) \mathbf{H}_{0}+F\left(\mathbf{H}_{0}+\mathbf{H}^{\prime}\right)=\mathbf{H}_{0}+F \mathbf{H}^{\prime} .
$$

Taking into account that $\mathbf{H}^{\prime}=0$ outside SRR, from the boundary conditions we obtain the relation

$$
\left|\mathbf{H}^{\prime}\right|=\frac{4 \pi}{c}\left|\mathbf{j}_{s}\right|,
$$

where $\mathbf{j}_{s}$ is an equivalent surface current in SRR, which is equal to the current per unit length. From Eq. (5) and 

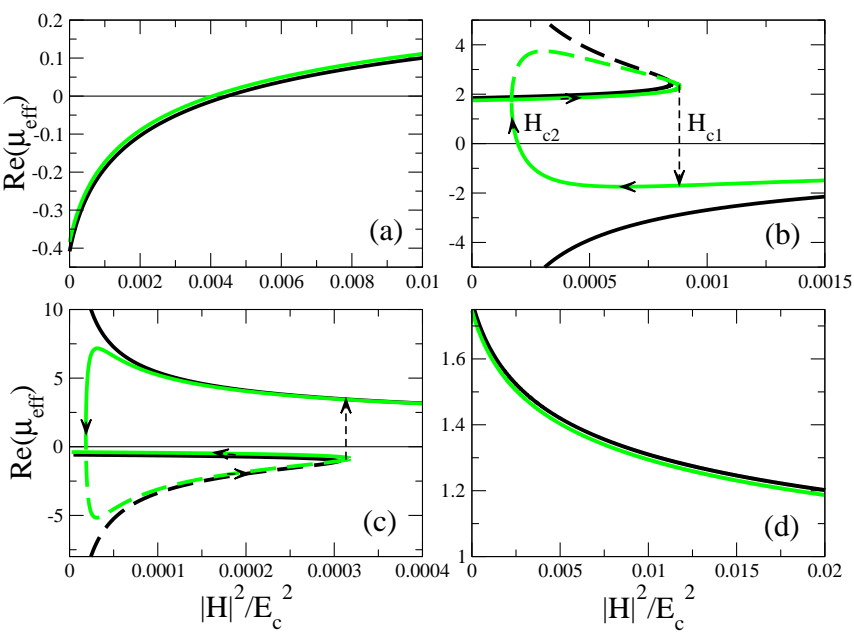

FIG. 2: Real part of the effective magnetic permeability vs. intensity of the magnetic field: (a) $\Omega>1, \alpha=1$; (b) $\Omega<1$, $\alpha=1$, (c) $\Omega>1, \alpha=-1$; and (d) $\Omega<1, \alpha=-1$. Black - the lossless case $(\gamma=0)$, grey-the lossy case $(\gamma=0.05)$. Dashed curves show unstable branches.

Eqs. (7)-(9), we obtain an explicit expression for the effective magnetic permeability of the composite structure (for $F \ll 1$ ):

$$
\mu_{\mathrm{eff}}(\mathbf{H})=1+\frac{F \omega^{2}}{\omega_{0 N L}^{2}(\mathbf{H})-\omega^{2}+i \Gamma \omega}
$$

where

$$
\omega_{0 N L}^{2}(\mathbf{H})=\left(\frac{c}{a}\right)^{2} \frac{d_{g}}{\left[\pi(1-F) h \epsilon_{D}\left(\left|\mathbf{E}_{g}(\mathbf{H})\right|^{2}\right)\right]}
$$

is the eigenfrequency of oscillations in the presence of the external field of a finite amplitude, $h$ is the width of the ring, $\Gamma=c^{2} / 2 \pi(1-F) \sigma a h$, for $h<\delta$, and $\Gamma=c^{2} / 2 \pi(1-F) \sigma a \delta$, for $h>\delta$. It is important to note that Eq. (10) has a simple physical interpretation: The resonant frequency of the artificial magnetic structure depends on the amplitude of the external magnetic field and, in turn, this leads to the intensity-dependent function $\mu_{\mathrm{eff}}$.

To be more specific, we consider the Kerr nonlinearity of the dielectric in the composite material, i.e

$$
\epsilon_{D}\left(|E|^{2}\right)=\epsilon_{D 0}+\alpha|E|^{2} / E_{c}^{2},
$$

where $E_{c}$ is a characteristic electric field, $\alpha= \pm 1$ stands for the focusing or defocussing nonlinearity, respectively. Then, the relation between the macroscopic magnetic field and the dimensionless nonlinear resonant frequency can be obtained from Eqs. (5)-(9), and (11) as

$$
|\mathbf{H}|^{2}=\alpha A^{2} E_{c}^{2} \frac{\left(1-X^{2}\right)\left[\left(X^{2}-\Omega^{2}\right)+\Omega^{2} \gamma^{2}\right]}{X^{6}},
$$

where $A^{2}=16 \epsilon_{D 0}^{3}(1-F) \omega_{0}^{2} h^{2} / c^{2}, \Omega=\omega / \omega_{0}, \omega_{0}=$ $(c / a)\left[d_{g} / \pi(1-F) h \epsilon_{D 0}\right]^{1 / 2}$ is the eigenfrequency of the
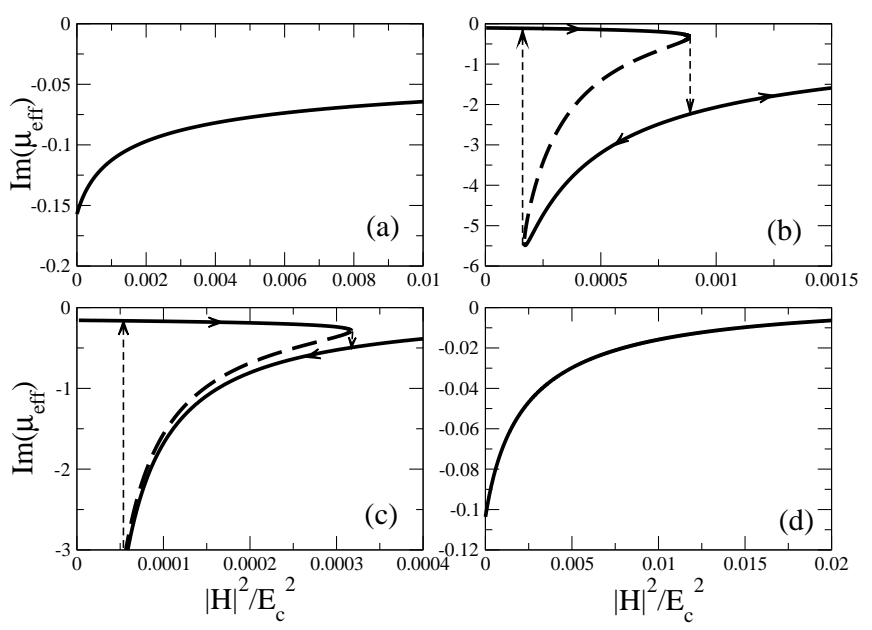

FIG. 3: Imaginary part of the effective magnetic permeability vs. intensity of the magnetic field for $\gamma=0.05$ : (a) $\Omega>1$, $\alpha=1$; (b) $\Omega<1, \alpha=1$, (c) $\Omega>1, \alpha=-1$; and (d) $\Omega<1$, $\alpha=-1$. Dashed curves show unstable branches.

system of SRRs in the linear limit, $X=\omega_{0 N L} / \omega_{0}$, and $\gamma=\Gamma / \omega_{0}$. Therefore, we find that the dimensionless eigenfrequency of the SRR lattice $X\left(|H|^{2}\right)$ is a multivalued function of the magnetic field. This result reveals a general property of nonlinear oscillators with a high quality factor [1].

Parametric dependence of the effective magnetic permeability on the magnetic field is determined completely by Eqs. (12) and (10). Figures 2 and 3 summarize different types of nonlinear magnetic properties of the composite, which are defined by the dimensionless frequency of the external field $\Omega$, for both focusing [Fig. $2(\mathrm{a}, \mathrm{b})$ and Fig. [3(a,b)] and defocussing [Fig. 2( c,d) and Fig. B (c,d)] nonlinearity of the dielectric.

In the case of focusing nonlinearity (i.e. when $\alpha=1$ ), the SRR eigenfrequency decreases with the intensity of the electromagnetic field because of a growth of the SRR capacitance. Then, for $\Omega>1$, the effective magnetic permeability of the composite structure grows with the field intensity, as shown in Fig. 22 a). If in the linear limit the composite material is left-handed, i.e. $\operatorname{Re}\left(\mu_{\mathrm{eff}}\right)<0$, it will become right-handed for higher intensities of the magnetic field [in this reasoning we assume $\operatorname{Re}\left(\epsilon_{\mathrm{eff}}\right)<0$ ].

More complicated behavior of the magnetic permeability is observed for $\Omega<1$; this is shown in Fig. 2(b). Here, in the linear limit the real part of the magnetic permeability is always positive, but the eigenfrequency of SRR decreases with the growth of the magnetic field, thus driving the system into the resonance. Since a nonlinear oscillator has a hysteresis structure of its response with a change of an external force (see, e.g., Ref. [1]), this leads to multi-valued dependencies. In our problem, the nonlinear eigenfrequency is a three-valued function of the external magnetic field, and this results in jumps of the magnetic permeability with the growth of the magnetic field. As follows from Fig. 2(b), the mag- 
netic field intensity displays a jump of the magnetic permeability from positive to negative values at some $H_{c 1}$. Thus, the initially opaque medium with positive refraction becomes a negative-refraction transparent medium with the growth of the field intensity. This effect can be treated as the second-order phase transition induced by the external electromagnetic field. Reversed transition takes place when the magnetic field intensity decreases to the value $H_{c 2}<H_{c 1}$.

In the case of defocussing nonlinearity (i.e. when $\alpha=-1$ ), the SRR eigenfrequency increases with the amplitude of the external field. That is why the resonance effects take place for $\Omega>1$, as shown in Figs. 2(c,d) and Figs. 3( c,d). Here, we observe the opposite behavior when the transition from the case $\operatorname{Re}\left(\mu_{\mathrm{eff}}\right)<0$ to the case $\operatorname{Re}\left(\mu_{\text {eff }}\right)>0$ takes place at high values of the external field, and the reversed transition occurs at lower field intensities. In the latter case, $\operatorname{Re}\left(\mu_{\text {eff }}\right)$ is always positive for $\Omega<1$, see Fig. [2 d).

Our results show that the imaginary part of the effective magnetic permeability, which determines the structure losses, can be controlled rather effectively by a proper choice of the intensity of the external highfrequency magnetic field, see Fig. 3. We believe that this feature may be important for the future applications of left-handed materials.

Due to the high values of the electric field in the gap of SRR and the resonant interaction of the electromagnetic field with the SRR lattice, the characteristic magnetic nonlinearity in such structures is much stronger then the corresponding electric nonlinearity. Therefore, the magnetic nonlinearity should dominate in the composite materials which display the phenomenon of negative refraction. Moreover, nonlinear elements can be used only for filling the gap in SRR, allowing an easy tuning by applying an external field.

The possibility of strongly enhanced nonlinearities in left-handed metamaterials revealed here may lead to an essential revision of the concepts based on the linear theory, since the electromagnetic waves propagating in such materials always have a finite amplitude. At the same time, the engineering of highly nonlinear composite materials will open a number of novel opportunities for their microwave applications, such as frequency multipliers, beam spatial spectrum transformers, switchers, limiters, etc.

It is important to note that the hysteresis behavior with jumps in the dependencies of the effective material parameters has been described above for the stationary processes only. Such transitions will display a characteristic scale in time or space for initial or boundary problems, respectively. Such spatial or temporal scales are determined by the relaxation micro-processes in the SRR lattice.

In conclusion, we have presented, for the first time to our knowledge, a systematic analysis of nonlinear properties of the microstructured materials which display negative refraction, the so-called left-handed metamaterials. We have shown that the composite metamaterials composed of a lattice of wires and split-ring resonators possess an effective magnetic permeability which depends on the intensity of the macroscopic magnetic field in a nontrivial way. The magnetic nonlinearity is found to be much stronger than the nonlinearity in the dielectric properties due to the field enhancement in the split-ring resonators. The dependence of the effective magnetic permeability on the field intensity allows switching between its positive and negative values, i.e. a change of the material properties from left- to right-handed and back. Such processes can be treated as the second-order phase transitions induced by the variation of an external electromagnetic field.

We thanks Costas Soukoulis for useful discussions and suggestions. This work was partially supported by the Australian Research Council and the US Air Force-Far East Office.
[1] J.B. Pendry, A.J. Holden, W.J. Stewart, and I. Youngs, Phys. Rev. Lett. 76, 4773 (1996).

[2] J. B. Pendry, A. J. Holden, D. J. Robbins, and W. J. Stewart, IEEE Trans. Microwave Theory Tech. 47, 2075 (1999).

[3] P. Markos and C.M. Soukoulis, Phys. Rev. E 65, 036622(2002); Phys. Rev. B 65, 033401 (2002).

[4] D.R. Smith, W. Padilla, D.C. Vier, S.C. Nemat-Nasser, and S. Shultz, Phys. Rev. Lett. 84, 4184 (2000).

[5] M. Bayindir, K. Aydin, E. Ozbay, P. Markos, and C. M. Soukoulis Appl. Phys. Lett. 81, 120 (2002).

[6] C.G. Parazzoli, R.B. Greegor, K. Li, B.E.C. Koltenbah, and M. Tanielian, Phys. Rev. Lett. 90, 107401 (2003).
[7] V. G. Veselago, Sov. Phys. Uspekhi 8, 2854 (1967) [Sov. Phys. Usp. 10, 509 (1968)].

[8] C. Luo, S.G. Johnson, and J. D. Joannopoulos, Appl. Phys. Lett. 83, 2352 (2002).

[9] C. Luo, S.G. Johnson, J.D. Joannopoulos, J.B. Pendry, Phys. Rev. B 65, 201104(R) (2002).

[10] M. Gorkunov, M. Lapine, E. Shamonina, and K.H. Ringhofer, Eur. Phys. J. B 28, 263 (2002).

[11] M.I. Rabinovich and D.I. Trubetskov, Oscillations and Waves in Linear and Nonlinear Systems (Dordrecht, Kluwer, 1989). 